\title{
A COLANGIOPANCREATOGRAFIA RETRÓGRADA ENDOSCÓPICA PODE SER REALIZADA COM SEGURANÇA EM CARÁTER AMBULATORIAL
}

\author{
Mara Virginia Lellis MARÇAL, Fernanda Prata Borges Martins THULER e Angelo Paulo FERRARI
}

RESUMO - Racional - A colangiopancreatografia endoscópica retrógrada é técnica efetiva no manejo das doenças biliopancreáticas. A segurança da realização do exame em ambulatório tem sido alvo de estudo. Objetivo - Avaliar a segurança da realização da colangiopancreatografia endoscópica retrógrada em ambulatório e descrever as complicações do exame. Casuística e Método-Acompanharam-se, prospectivamente, pacientes ambulatoriais encaminhados para colangiopancreatografia endoscópica retrógrada durante o período de 2001 a 2003 . Complicações foram definidas segundo critérios de consenso, incluindo todos os efeitos adversos relacionados ao exame. Resultados - Foram incluídas 195 colangiopancreatografias endoscópicas retrógradas, 79 (40,5\%) diagnósticas e $116(59,5 \%)$ terapêuticas. O grupo incluiu 112 mulheres, com média de idade de 51 anos $( \pm 18,9)$. Os diagnósticos encontrados mais freqüentemente foram: cálculo biliar $(30,2 \%)$, estenose benigna $(13,8 \%)$, neoplasia $(10,2 \%)$ e pancreatite crônica $(10,2 \%)$. Obteve-se sucesso em $88,6 \%$ dos exames diagnósticos e $78,5 \%$ dos terapêuticos. Dos 195 pacientes, $10(5,1 \%)$ necessitaram de observação, dentre os quais $7(3,6 \%)$ foram internados, $(2$ pacientes com pancreatite aguda, 2 com perfurações, 1 com hemorragia, 1 com complicação cardiorespiratória e 1 com febre). Dos 188 casos liberados após o exame, 8 $(4,2 \%)$ foram readmitidos ( 1 pancreatite aguda, 1 hemorragia, 1 perfuração, 3 colangite, 2 dor abdominal). Ao comparar o grupo das complicações identificadas imediatamente contra o segundo, não se encontrou diferença estatisticamente significante quanto à idade, sexo, diagnóstico e/ou grau de dificuldade do exame. Conclusão - O tamanho da amostra e os resultados negativos da análise estatística impediram a determinação de fatores de risco, independentes para complicações pós- colangiopancreatografia endoscópica retrógrada. Contudo, não houve nenhum óbito ou complicações com má evolução nos pacientes inicialmente liberados, confirmando a segurança na realização da colangiopancreatografia endoscópica retrógrada em ambulatório.

DESCRITORES - Pancreatocolangiografia retrógada endoscópica. Pacientes ambulatoriais.

\section{INTRODUÇÃO}

A colangiopancreatografia endoscópica retrógrada (CPRE) é uma das técnicas mais efetivas no manejo das doenças pancreatobiliares, seja para diagnóstico ou realização de terapêutica.

Alguns estudos têm sido realizados com objetivo de avaliar a segurança do procedimento quando realizado em ambulatório, enfatizando a realização de procedimentos terapêuticos ${ }^{(3,4,5,6}$, $7,9,11,12,15)$. As complicações relacionadas ao exame podem ser mais bem avaliadas pelos estudos prospectivos, uma vez que os sintomas das complicações podem aparecer apenas 12-24 horas após o procedimento ${ }^{(4,5,6,7,9)}$

O objetivo deste estudo foi avaliar a segurança da CPRE diagnóstica e terapêutica realizada em regime ambulatorial e descrever as complicações relacionadas ao procedimento.

\section{CASUÍSTICA E MÉTODO}

Avaliaram-se, prospectivamente, 195 pacientes de ambulatório encaminhados ao Setor de Endoscopia do Hospital São Paulo - Universidade Federal de São Paulo, São Paulo, SP, para realização de CPRE no período de 2001 a 2003.

O trabalho foi aprovado pelo Comitê de Ética em Pesquisa da instituição. Cada paciente recebeu e assinou termo de consentimento, sendo informado sobre o procedimento e suas possíveis complicações.

A CPRE foi realizada sob sedação individualizada, com midazolam, diazepam, propofol e meperidina, associados ou não. Antibioticoterapia profilática foi realizada quando indicada.

Em pacientes com menos de 70 anos e sem outras doenças graves a sedação era inicialmente feita com $100 \mathrm{mg}$ de meperidina e $3 \mathrm{mg}$ de midazolan ou $10 \mathrm{mg}$ de diazepam. De acordo com

Disciplina de Gastroenterologia, Universidade Federal de São Paulo, Escola Paulista de Medicina - UNIFESP-EPM, São Paulo, SP.

Endereço para correspondência: Dr. Angelo Paulo Ferrari - Rua Machado Bittencourt, 379 - apto 91 - 04044-001 - São Paulo, SP. E-mail: angelo@gastro.epm.br 
necessidades individuais, eram feitos reforços com doses pequenas (2 $\mathrm{mg}$ de midazolam, 25 a $50 \mathrm{mg}$ de meperidina, ou $5 \mathrm{mg}$ de diazepam). $\mathrm{O}$ reforço da dose era feito até sedação suficiente para realização do exame ou desaturação detectada por oximetria de pulso. A partir da segunda metade do estudo, nos pacientes em que não se conseguiu bom nível de sedação, procedeu-se à associação com propofol (não utilizado em pacientes com cardiomiopatias). Propofol é droga anestésica de rápido pico de ação e rápida metabolização, que age nos receptores centrais do ácido gama-aminobutírico (GABA). Necessita de maior cuidado na monitorização, já que freqüentemente leva a queda da saturação de hemoglobina, o que pode ser potencializado quando outras drogas estão associadas. Não há droga descrita para reversão do efeito.

Em pacientes idosos ou com outras doenças graves, as doses eram diminuídas ou em certos casos, avaliadas individualmente, sendo o exame realizado com o auxílio de anestesista.

Após o exame, os pacientes permaneceram em observação por um período mínimo de 2 horas. Na ausência de evidências de complicações, tais como dor persistente, náuseas, vômitos, febre, instabilidade dos sinais vitais ou sedação prolongada, foram dispensados para casa. Quando se suspeitou de complicação, os pacientes foram mantidos em observação por período mais prolongado ou internados, se necessário. Todos foram reavaliados por contato telefônico num prazo de 24 horas e 30 dias após o exame para possíveis sinais e sintomas de complicações.

Foram consideradas complicações associadas à CPRE: pancreatite, hemorragia, colangite, perfuração, complicações cardiorespiratórias, e também dor ou febre pós-procedimento. Pancreatite aguda foi definida como dor abdominal e/ou vômitos acompanhados por elevação da amilase sérica pelo menos 3 vezes o limite superior de normalidade, 24 horas após o procedimento ${ }^{(2)}$. Hemorragia foi definida como evidência clínica de sangramento, com sintomas ou alteração hemodinâmica ${ }^{(2)}$. Colangite foi diagnosticada quando o paciente apresentou febre $\geq$ $38,5^{\circ} \mathrm{C}$, por mais de 24 horas, com hemocultura positiva ${ }^{(2)}$. Perfuração retroperitonial ou em cavidade livre foi considerada quando documentada por exame radiológico ${ }^{(2)}$.

As complicações foram descritas e avaliadas quanto à distribuição por sexo, idade, diagnóstico e grau de dificuldade do exame.

Readmissão hospitalar após a liberação inicial foi considerada uma complicação quando o evento preencheu os critérios acima.

O grau de dificuldade do exame foi determinado segundo a classificação de SCHUTZ e ABBOTT ${ }^{(11)}$, reproduzida no Quadro 1.

A análise estatística dos dados foi realizada através do teste de Mann-Whitney e teste de comparação de proporções com o programa "Primer of Biostatistics". O resultado foi considerado estatisticamente significativo quando valor de $P$ encontrado foi $<0,05$.

\section{RESULTADOS}

Do total das 195 CPRE estudadas, 79 exames (40,5\%) foram diagnósticos e $116(59,5 \%)$ terapêuticos. O grupo foi constituído por 112 pacientes do sexo feminino e 83 do masculino, com média de idade de $51( \pm 18,9)$ anos.

Dentre os diagnósticos encontrados, cálculo biliar foi o mais freqüente, respondendo por $30,2 \%$ dos casos (59 pacientes), estenose benigna foi o segundo acometendo $13,8 \%$ da amostra (27), seguido por neoplasia $10,2 \%$ (20), pancreatite crônica $10,2 \%$ (20) e outros diagnósticos em 5,1\% (10) dos casos (Tabela 1).
QUADRO 1 - Classificação de SCHUTZ e ABBOTT ${ }^{(13)}$ quanto ao grau de dificuldade da colangiopancreatografia endoscópica

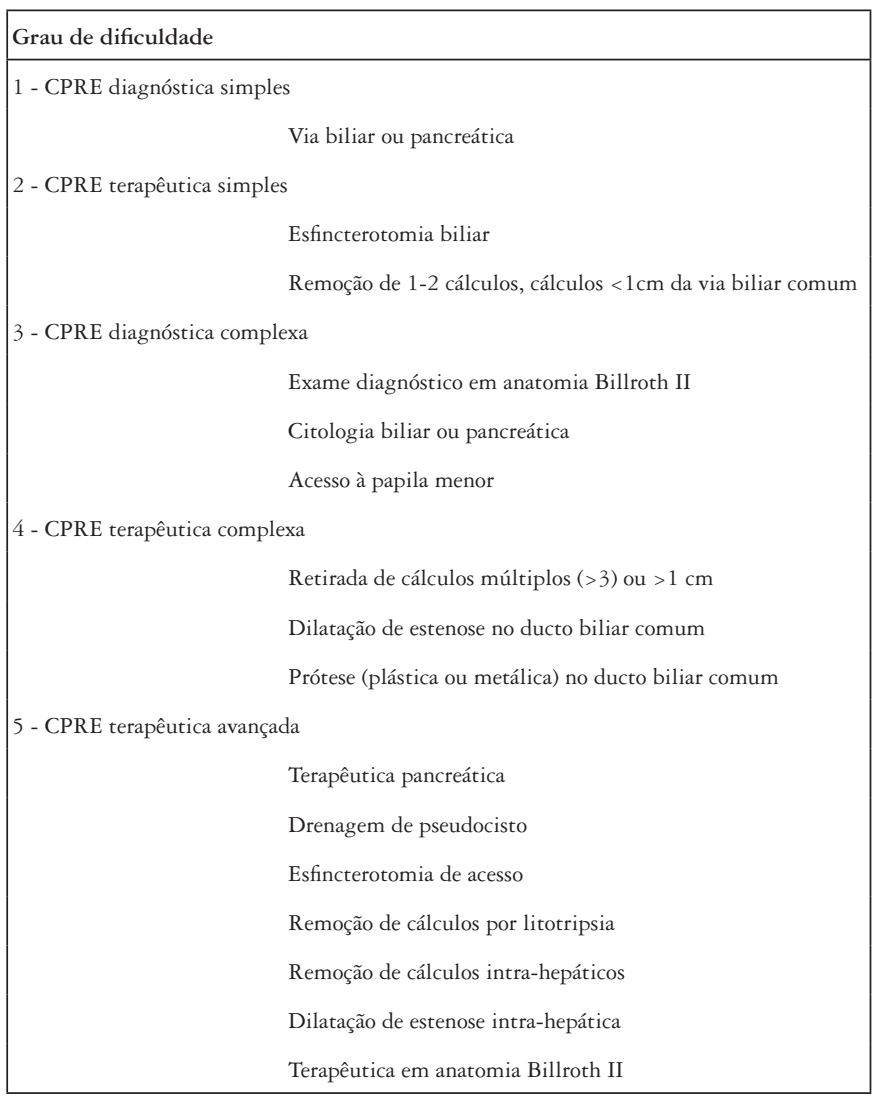

TABELA 1 - Distribuição dos exames quanto ao diagnóstico, sexo e idade dos pacientes

\begin{tabular}{lccc}
\hline Diagnóstico & $\begin{array}{c}\text { Complicações } \\
\text { diagnosticadas } \\
\text { imediatamente }\end{array}$ & $\begin{array}{c}\text { Complicações } \\
\text { diagnosticadas } \\
\text { tardiamente }\end{array}$ & Sem complicações \\
\hline Normal & $1(0,5 \%)$ & $1(0,5 \%)$ & $57(29,3 \%)$ \\
Cálculo & $3(1,5 \%)$ & $3(1,5 \%)$ & $53(27,2 \%)$ \\
Estenose benigna & $1(0,5 \%)$ & $3(1,5 \%)$ & $23(11,8 \%)$ \\
Neoplasia & - & - & $20(10,2 \%)$ \\
Pancreatite crônica & $4(2,0 \%)$ & - & $16(8,2 \%)$ \\
Outros & $1(0,5 \%)$ & $1(0,5 \%)$ & $8(4,1 \%)$ \\
\hline Sexo (M:F) & $6: 4$ & $1: 7$ & $76: 101$ \\
\hline Idade (anos) & $37,3( \pm 17,1)$ & $53,5( \pm 19,3)$ & $52( \pm 18,6)$ \\
\hline
\end{tabular}

Os exames diagnósticos foram bem sucedidos em 88,6\% (70/79), enquanto a realização da terapêutica proposta foi alcançada em 78,5\% (91/119). As principais causas de insucesso do procedimento foram falha de acesso à via biliar ou pancreática e falha da terapêutica, apesar do acesso, respondendo por $58,8 \%$ e $32,4 \%$, respectivamente.

Dentre os 37 insucessos, a sedação foi responsável por 3 casos $(8,8 \%)$. Em um houve depressão cardiorespiratória e o exame foi interrompido. Nos outros dois, na metade pré-propofol, não foi conseguida sedação suficiente para realização do procedimento. 
A taxa total de complicações encontrada na amostra da presente série foi de $9,2 \%$ (18 casos), das quais 4 ( $2 \%$ ) ocorreram em exames diagnósticos e $14(7,2 \%)$ em terapêuticos. A Tabela 2 apresenta a distribuição das complicações de acordo com a realização ou não da terapêutica. Observaram-se quatro $(2,05 \%)$ de complicações severas.

TABELA 2 - Distribuição das complicações diagnosticadas imediata e tardiamente com respeito à realização ou não de procedimento terapêutico

\begin{tabular}{lcccc}
\hline Complicações & \multicolumn{2}{c}{ CPRE diagnóstica } & \multicolumn{2}{c}{ CPRE terapêutica } \\
\hline & $\begin{array}{c}\text { Complicações } \\
\text { diagnosticadas } \\
\text { imediatamente }\end{array}$ & $\begin{array}{c}\text { Complicações } \\
\text { diagnosticadas } \\
\text { tardiamente }\end{array}$ & $\begin{array}{c}\text { Complicações } \\
\text { diagnosticadas } \\
\text { imediatamente }\end{array}$ & $\begin{array}{c}\text { Complicações } \\
\text { diagnosticadas } \\
\text { tardiamente }\end{array}$ \\
\hline Pancreatite aguda & $1(0,5 \%)$ & - & $1(0,5 \%)$ & $1(0,5 \%)$ \\
Hemorragia & - & - & $1(0,5 \%)$ & $1(0,5 \%)$ \\
Colangite & - & $1(0,5 \%)$ & - & $2(1,0 \%)$ \\
Perfuração & - & - & $2(1,0 \%)$ & $1(0,5 \%)$ \\
Cardiorespiratórias & - & - & $1(0,5 \%)$ & - \\
Dor ou febre & $1(0,5 \%)$ & $1(0,5 \%)$ & $3(1,5 \%)$ & $1(0,5 \%)$ \\
\hline
\end{tabular}

Dez pacientes $(5,1 \%)$ necessitaram de observação mais prolongada após o procedimento. Dentre estes, sete $(3,6 \%)$ foram internados por suspeita de complicação, confirmada em todos os casos (dois por pancreatite aguda, dois por perfurações, um por hemorragia, um por complicação cardiorespiratória e um por febre). Os outros três que haviam apresentado respectivamente dor, alergia no local da venopunção e tremores após o término do exame, foram dispensados, sem necessidade de posterior reinternação (Tabela 2 ).

Dos 188 casos dispensados após o exame, 8 (4,2\%) foram readmitidos no hospital por complicações (Tabela 2): pancreatite aguda (1), hemorragia (1), perfuração (1), colangite (3) e dor abdominal (2).

As complicações foram distribuídas também quanto ao grau de dificuldade do exame (Tabela 3).

TABELA 3 - Distribuição das complicações segundo o grau de dificuldade do exame

\begin{tabular}{cccc}
\hline $\begin{array}{c}\text { Grau de } \\
\text { dificuldade }\end{array}$ & $\begin{array}{c}\text { Complicações } \\
\text { diagnosticadas } \\
\text { imediatamente }\end{array}$ & $\begin{array}{c}\text { Complicações } \\
\text { diagnosticadas } \\
\text { tardiamente }\end{array}$ & Total de exames \\
\hline 1 & $2(2,9 \%)$ & $2(2,9 \%)$ & 69 \\
2 & $3(11,5 \%)$ & $1(3,8 \%)$ & 26 \\
3 & - & - & 3 \\
4 & - & $3(6,7 \%)$ & 45 \\
5 & $5(9,6 \%)$ & $2(3,8 \%)$ & 52 \\
\hline
\end{tabular}

Comparou-se o grupo de pacientes em que as complicações foram identificadas imediatamente após o exame, com o grupo em que elas foram diagnosticadas tardiamente, necessitando readmissão hospitalar. Não se encontrou diferença estatisticamente significativa quanto à idade, sexo ou diagnóstico do exame. Analisou-se, também, a distribuição das complicações quanto ao grau de dificuldade do exame, igualmente sem diferença estatística.

O acompanhamento dos pacientes por 30 dias permite a identificação de complicações tardias; porém, além das já citadas, encontraram-se apenas complicações relacionadas à prótese.

\section{DISCUSSÃO}

Apesar das variações dos formatos dos trabalhos que estudam as complicações da CPRE, as taxas relatadas variam em torno de $8 \%{ }^{(1,}$ 2, 6, 7, 9, 10,11, 12, 15) Estudos prospectivos tendem a demonstrar taxas de complicações maiores e a reavaliação em 30 dias possibilita o diagnóstico de complicações tardias. A taxa de complicações encontrada nesta casuística de exames ambulatoriais foi de 9,2\%, semelhante aos dados previamente relatados na literatura.

A maioria dos estudos existentes sobre a realização de CPRE terapêutica ambulatorial sugere período de observação de 2 a 3 horas após o exame ${ }^{(6,15)}$, baseados no fato de que a maior parte das complicações manifesta-se neste intervalo. Entretanto, FREEMAN et al. ${ }^{(7)}$ observaram que nas primeiras 2 horas, apenas $44 \%$ dos pacientes que complicaram desenvolveram sintomas e em 6 horas $79 \%$ tinham sintomas.

FREEMAN et al. ${ }^{(7)}$ realizaram estudo prospectivo, multicêntrico, com análise multivariada de possíveis fatores preditores de readmissão hospitalar por complicações em 614 pacientes submetidos a CPRE em regime ambulatorial. Após observação inicial e liberação, 35 doentes $(5,7 \%)$ foram readmitidos por complicações (20 por pancreatite aguda e 15 por outras). A análise mostrou serem fatores independentes preditores de reinternação, os seguintes: disfunção do esfíncter de Oddi, cirrose, canulação difícil do ducto biliar, pré-corte e exame percutâneo combinado, aumentando em 3 vezes a chance de readmissão. Concluíram que a CPRE é procedimento amplamente utilizado e relativamente seguro, resultando porém, em grande número de reinternações por complicações, sugerindo que pacientes de alto risco ou com pelo menos um dos cinco fatores independentes devam ser observados por, no mínimo, 6 horas.

METHA et al. ${ }^{(11)}$ avaliaram, retrospectivamente, dados de 262 CPRE realizadas em ambiente ambulatorial, excluídos casos onde foi realizada manometria e/ou qualquer intervenção pancreática, encontraram taxa de complicação em 30 dias de 5,7\% (15 casos). Nove pacientes ( $3,4 \%)$ necessitaram de internação, dos quais sete apresentavam sintomas nas primeiras 4 horas e foram internados logo após o procedimento: cinco por pancreatite aguda, um por hemorragia, um por perfuração; os outros dois foram liberados e posteriormente readmitidos por hemorragia e colangite. Esses autores sugerem, que 4 horas seja um intervalo de tempo adequado para observação após a CPRE, concluindo que esfincterotomia e colocação de prótese biliar podem ser realizadas nesse espaço com segurança.

Estudo brasileiro realizado por BALBINOTTI et al. ${ }^{(1)}$ avaliou prospectivamente as complicações da CPRE em 311 pacientes, dos quais $63 \%$ terapêuticos. Observaram taxa de complicação de $8,4 \%$, sendo pancreatite aguda a mais freqüente, respondendo por $5,1 \%$ dos casos. Não foi objetivo do estudo analisar separadamente exames realizados no ambiente em questão e taxa de reinternação por complicação.

THAM et al. ${ }^{(15)}$ revisaram os dados de 190 CPRE terapêuticas realizadas e, ambulatório. Internação hospitalar foi necessária em 31 casos $(16 \%), 22$ por complicações $(11,6 \%)$ e $9(4,4 \%)$ para observação. Dos 31 pacientes internados, 26 (13\%) foram admitidos após a CPRE e $5(3 \%)$ liberados após o exame e retornaram ao hospital após intervalo médio de 24 horas, sendo pancreatite aguda a complicação mais freqüente. Concluíram que essa modalidade de CPRE terapêutica realizada em grupo selecionado de pacientes, 
permanecendo em observação após o exame por período de 2 horas e internação reservada para aqueles com suspeita de complicação, parece ser segura e reduzir custos.

HO et al ${ }^{(9)}$ estudaram prospectivamente 415 pacientes submetidos a CPRE terapêutica ambulatorial. Internação hospitalar foi necessária para $41(9,9 \%)$ pacientes por complicações e $63(15,2 \%)$ para observação após o exame. Dos 41 internados por complicações pós-CPRE, 29 foram admitidos logo após o procedimento e os demais readmitidos após liberação inicial. A análise multivariada dos dados sugere que sejam fatores de risco para internação por mais de 1 dia: dor durante o procedimento, história de pancreatite e realização de esfincterotomia. A presença dos três fatores esteve associada a $67 \%$ de probabilidade de internação. Concluem, que esses três sejam fatores independentes preditores de internação hospitalar.

ELFANT et al.(6) estudaram prospectivamente 98 pacientes ambulatoriais encaminhados para CPRE terapêutica por coledocolitíase, em $94,9 \%$ dos casos (93) foi realizada esfincterotomia. Os pacientes foram observados por período de 1 a 3 horas e então liberados para casa. Um paciente ficou internado por hemorragia pós-esfincterotomia e dois $(2,1 \%)$ foram readmitidos por pancreatite aguda 24 horas após a alta. Esses autores concluíram que a terapia endoscópica para coledocolitíase pode ser realizada com segurança em pacientes ambulatoriais, com redução de custos.

Pancreatite aguda é a complicação mais freqüentemente associada

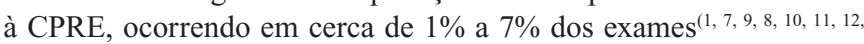
15). Foi também uma das principais complicações na presente série, ocorrendo em 1,5\% dos exames. Dos três casos de pancreatite aguda, um não foi identificado no período de observação após o exame. O atraso no aparecimento dos sintomas pode estar associado à sedação e analgesia, ou à demora de fato do início da dor.

Hemorragia é complicação mais diretamente relacionada à realização da esfincterotomia, com incidência relatada de $0,76 \%$ a $2,5 \%{ }^{(8,10)}$ Na metade dos casos a manifestação do sangramento pode ser tardia, ocorrendo dias após o procedimento ${ }^{(7,11,15)}$. Na presente série, encontrou-se taxa de $1,0 \%$ de hemorragia. Em um dos dois casos, o sangramento não foi detectado durante o procedimento e o doente foi readmitido 4 dias após a esfincterotomia.

Outras complicações como colangite e colecistite aguda, geralmente manifestam-se 24 horas após o procedimento ${ }^{(7,10)}$. Como foi observado nesta casuística, os três pacientes $(1,5 \%)$ que desenvolveram colangite haviam sido liberados após o exame e foram readmitidos posteriormente para tratamento da complicação.

A ocorrência de perfuração durante o exame tem sido relatada em até $1 \%$ dos casos após esfincterotomia, geralmente retroperitonial, exceto em alterações anatômicas ${ }^{(6,10,14)}$. Neste estudo encontraram-se três casos de perfuração $(1,5 \%)$, dois deles associados à esfincterotomia e um após punção de pseudocisto pancreático.

As complicações cardiorespiratórias são mais raras, ocorrendo em menos de $1 \%$ dos casos. Geralmente são decorrentes da sedação utilizada ou da doença de base ${ }^{(8,10)}$. Observou-se um caso $(0,5 \%)$ de depressão respiratória pelos sedativos.

No presente estudo, analisaram-se os diversos procedimentos da CPRE, estudados separadamente nos trabalhos anteriormente descritos, através do grau de dificuldade dos exames, porém não se encontrou diferença estatisticamente significante quanto à taxa de complicações e os diferentes graus de dificuldade do mesmo.

Assim como nos estudos referidos anteriormente, observou-se maior taxa de complicações nos exames terapêuticos. Embora não haja dados publicados que permitam esta conclusão, parece que neles, principalmente nos com maior grau de complexidade, o tempo de observação após o procedimento deva ser prolongado (talvez maior do que 2 horas). Além disso, em nosso meio, provavelmente seja importante investigar a facilidade do paciente (no que se relaciona a acesso a transporte) em retornar ao Serviço ou procurar outro local de atendimento médico na evidência da ocorrência de qualquer sinal ou sintoma de complicação.

\section{CONCLUSÃO}

O tamanho da amostra estudada e os resultados negativos encontrados na análise estatística impediram a determinação de fatores de risco independentes para complicações pós-CPRE. Observou-se, contudo, que o índice de complicações ocorridas nos procedimentos ambulatoriais é semelhante àquele dos exames realizados durante internação hospitalar. A metade delas pôde ser identificada logo após o procedimento e a outra metade identificada tardiamente, porém sem determinar evolução negativa do caso, sem ocorrência de nenhum óbito.

Marçal MVL, Thuler FPBM, Ferrari AP. Safety of endoscopic retrograde cholangiopancreatography performed in ambulatory centers. Arq Gastroenterol 2004;42(1):4-8

ABSTRACT - Background - Endoscopic retrograde cholangiopancreatography is effective technique to approach various biliary and pancreatic disorders. Safety of endoscopic retrograde cholangiopancreatography on an outpatient basis has been questioned. Objectives - To evaluate the safety of outpatient endoscopic retrograde cholangiopancreatography and describe procedure complications. Patients/Method - We prospectively assessed outpatients endoscopic retrograde cholangiopancreatography during 2001 to 2003 period. Complications were defined according to consensus criteria and all adverse effects related to procedure were included. Results - One hundred and ninety five outpatients endoscopic retrograde cholangiopancreatography were performed, 79 ( $40.5 \%)$ diagnostic and $116(59.5 \%)$ therapeutic. The study group included 112 women, mean age $51( \pm 18.9)$ years. The most common diagnoses were: biliary calculi (30.2\%), benign stenosis, (13.8\%), malignant obstruction (10.2\%) and chronic pancreatitis (10.2\%). Success was achieved in $88.6 \%$ of diagnostic endoscopic retrograde cholangiopancreatography and $78.5 \%$ in therapeutic. Complications necessitating observation developed in $10(5.1 \%)$ of 195 endoscopic retrograde cholangiopancreatography, among them, 7 (3.6\%) were hospitalized, ( 2 acute pancreatitis, 2 perforations, 1 bleeding, 1 cardio-respiratory e 1 fever). Among 188 patients initially discharged $8(4.2 \%)$ needed readmission ( 1 acute pancreatitis, 1 bleeding, 1 perforation, 3 cholangitis, 2 abdominal pain). Comparing the first group where complications were immediately identified to the second, no significant statistical difference concerning to age, sex, diagnoses and procedure difficulty degree was found. Conclusion - Sample size and negative statistical results failed to determinate independent risk factors for outpatients endoscopic retrograde cholangiopancreatography complications. However, there were no deaths or bad evolution in patients released after examination, confirming the safety of outpatient endoscopic retrograde cholangiopancreatography.

HEADINGS - Cholangiopancreatography, endoscopic retrograde. Outpatients. 


\section{REFERÊNCIAS BIBLIOGRÁFICAS}

1. Balbinotti RA, Braga DC, Balbinotti SS, Boniatti MM, Bertuol NR, Batalha Jr MG, Pimentel F. Análise das complicações após realização de colangiopancreatografias retrógradas endoscópicas (CPRE). GED Gastroenterol Endosc Dig 2003;22:181-4.

2. Cotton PB, Lehman G, Vennes J, Geenen JE, Russell RC, Meyers WC, Liguory C Nickl N. Endoscopic sphincterotomy complications and their management: an attempt at consensus. Gastrointest Endosc 1991;37:383-93.

3. Cvetkovski B, Gerdes H, Kurtz RC. Outpatient therapeutic ERCP with endobiliary stent placement for malignant common bile duct obstruction. Gastrointest Endosc 1999;50:63-6.

4. Elfant AB, Alhalel R, Bourke MJ, Kortan P, Haber GB. Outpatient biliary stone extraction (BSE): a prospective study [abstract]. Gastrointest Endosc 1995;41:319.

5. Elfant AB, Bourke MJ, Alhalel R, Kortan P, Haber GB. Outpatient biliary endoprosthesis insertion (BEI): a prospective study [abstract]. Gastrointest Endosc 1995;41:319.

6. Elfant AB, Bourke MJ, Alhalel R, Kortan PP, Haber GB. A prospective study of the safety of endoscopic therapy for choledocholithiasis in an outpatient population. Am J Gastroenterol 1996;91:1499-502.

7. Freeman ML, Nelson DB, Sherman S, Haber GB, Fennerty MB, DiSario JA, Ryan ME, Kortan PP, Dorsher PJ, Shaw MJ, Herman ME, Cunningham JT, Moore JP, Silverman WB, Imperial JC, Mackie RD, Jamidar PA, Yakshe PN, Logan GM, Pheley AM. Same-day discharge after endoscopic biliary sphincterotomy: observations from a prospective multicenter complication study. The Multicenter Endoscopic Sphincterotomy (MESH) Study Group. Gastrointest Endosc 1999;49:580-6.
8. Freeman ML, DiSario JA, Nelson DB, Fennerty MB, Lee JG, Bjorkman DJ, Overby CS, Aas J, Ryan ME, Bochna GS, Shaw MJ, Snady HW, Erickson RV, Moore JP, Roel JP. Risk factors for post-ERCP pancreatitis: a prospective, multicenter study. Gastrointest Endosc 2001;54:425-34.

9. Ho KY, Montes H, Sossenheimer MJ, Tham TC, Ruymann F, Van Dam J, Carr-Locke DL. Features that may predict hospital admission following outpatient therapeutic ERCP. Gastrointest Endosc 1999; 49:587-92.

10. Loperfido S, Angelini G, Benedetti G, Chilovi F, Costan F, De Berardinis F, De Bernardin M; Ederle A; Fina P; Fratton A. Major early complications from diagnostic and therapeutic ERCP: a prospective multicenter study. Gastrointest Endosc 1998;48:1-10.

11. Mehta SN, Pavone E, Barkun AN. Outpatient therapeutic ERCP: a series of 262 consecutive cases. Gastrointest Endosc 1996;44:443-9.

12. Podolsky I, Kortan P, Haber GB. Endoscopic sphincterotomy in outpatients. Gastrointest Endosc 1989;35:372-6.

13. Schutz SM, Abbott RM. Grading ERCPs by degree of difficulty: a new concept to produce more meaningful outcome data. Gastrointest Endosc 2000;51(5):535-9.

14. Sherman S. Outpatient therapeutic ERCP: has the time come? Gastrointest Endosc 1997;45:326-8

15. Tham TC, Vandervoort J, Wong RC, Lichtenstein DR, Van Dam J, Ruymann F, Farraye F, Carr-Locke DL. Therapeutic ERCP in outpatients. Gastrointest Endosc 1997;45:225-30

Recebido em 9/2/2004 Aprovado em 22/6/2004 